\title{
The murine angiotensin II-induced abdominal aortic aneurysm model: rupture risk and inflammatory progression patterns
}

\author{
Richard Y. Cao' ${ }^{1}$ Tim St. Amand ${ }^{1}$, Matthew D. Ford ${ }^{2}$, Ugo Piomelli' ${ }^{2}$ and Colin D. Funk ${ }^{1 *}$ \\ Department of Physiology, Queen's University, Kingston, ON, Canada \\ 2 Department of Mechanical and Materials Engineering, Queen's University, Kingston, ON, Canada
}

Edited by:

Stefania Tacconelli, Università degli

Studi G. D'Annunzio, Italy

Reviewed by:

Emanuela Ricciotti, University of

Pennsylvania, USA

Miao Wang, University of Pennsylvania, USA

\section{*Correspondence:}

Colin D. Funk, Department of

Physiology, Queen's University, Stuart

Street, 433 Botterell Hall, Kingston, ON

K7L 3N6 Canada.

e-mail:funkc@queensu.ca
An abdominal aortic aneurysm (AAA) is an enlargement of the greatest artery in the body defined as an increase in diameter of 1.5-fold. AAAs are common in the elderly population and thousands die each year from their complications. The most commonly used mouse model to study the pathogenesis of AAA is the angiotensin II (Ang II) infusion method delivered via osmotic minipump for 28 days. Here, we studied the site-specificity and onset of aortic rupture, characterized three-dimensional (3D) images and flow patterns in developing AAAs by ultrasound imaging, and examined macrophage infiltration in the Ang II model using 65 apolipoprotein E-deficient mice. Aortic rupture occurred in 16 mice (25\%) and was nearly as prevalent at the aortic arch $(44 \%)$ as it was in the suprarenal region (56\%) and was most common within the first 7 days after Ang II infusion (12 of 16; 75\%). Longitudinal ultrasound screening was found to correlate nicely with histological analysis and AAA volume renderings showed a significant relationship with AAA severity index. Aortic dissection preceded altered flow patterns and macrophage infiltration was a prominent characteristic of developing AAAs. Targeting the inflammatory component of AAA disease with novel therapeutics will hopefully lead to new strategies to attenuate aneurysm growth and aortic rupture.

Keywords: inflammation, macrophage, ultrasound, apolipoprotein E, mouse model, pathogenesis

\section{INTRODUCTION}

The abdominal aortic aneurysm (AAA) is a localized dilatation of the abdominal aorta exceeding the normal diameter by more than $50 \%$ (Upchurch and Schaub, 2006). AAAs are associated with advanced age, male gender, cigarette smoking, atherosclerosis, hypertension, and a genetic predisposition (van Vlijmen-van Keulen et al., 2002; Golledge et al., 2006; Lloyd-Jones et al., 2010). The histopathologic features of AAAs are characterized by chronic inflammation in the vessel wall, tissue degeneration and remodeling, and depletion of medial smooth muscle cells (Thompson, 2002; McCormick et al., 2007). With an aging population, AAAs are becoming common vascular disorders with life-threatening implications such as aortic rupture, which has been reported to have a mortality rate as high as 90\% (Lloyd-Jones et al., 2010). AAAs account for more than 15,000 deaths/year in the United States (Baxter et al., 2008). Therefore, understanding the pathophysiology of AAAs and translating the knowledge from basic science to the clinical realm is certain to be a promising step to prevent this condition.

Since most information of human AAAs is obtained from pathological specimens after surgical intervention, there is a need to have animal models to mimic the entire progression sequence of aneurysm evolution. Several rodent models of AAA have been developed either via genetic exploitation or via chemical methods; their advantages and limitations have been considered (Daugherty and Cassis, 2004). The angiotensin II (Ang II)-induced mouse AAA model in the atherosclerotic-susceptible strain (apolipoprotein E deficient; $\mathrm{apoE}^{--}$) has become the most widely used model in recent years because of its simplicity and because certain facets of the model resemble human disease acquisition, including male gender preponderance in the setting of mild hypertension with enhanced incidence in the presence of hyperlipidemia (Daugherty et al., 2000, 2001; Saraff et al., 2003; Daugherty and Cassis, 2004). There is a well-defined time course of events in this model with early macrophage infiltration into the smooth muscle-rich medial layer of the aneurysm-prone area, transmedial dissection that causes rapid luminal expansion within the first 7 days of AngII infusion, with subsequent complex inflammatory events that include intramural thrombus formation, elastin degradation and profound remodeling in which the thrombus is often resorbed and replaced by fibrous tissue interspersed with leukocytes (Daugherty et al., 2000, 2006). However, there are still some parameters such as aortic rupture death and hemodynamical changes within the aorta upon aneurysm formation that are poorly defined. In this study, we attempt to reveal the inflammatory progression patterns during aneurysm formation, as well as elucidate the incidence and site-specificity of aortic rupture and follow in vivo aneurysmal development with $2 \mathrm{D}$ - and 3D-ultrasound imaging.

\section{MATERIALS AND METHODS MOUSE AAA MODEL INDUCTION}

ApoE ${ }^{-/-}$mice (backcrossed 10 times to the C57BL/6 background) used for this study were from the Jackson Laboratory (Bar Harbor, ME, USA). Genotyping was confirmed by PCR using tail clip samples as previously described (Cao et al., 2007). Alzet osmotic minipumps (Durect Corporation, Cupertino, CA, USA) loaded with Ang II (Sigma-Aldrich, Saint Louis, MO, USA) were implanted subcutaneously into $65 \mathrm{apoE}^{-/-}$male mice (8-12 weeks of age) in the dorsal region under isoflurane anesthesia to obtain a delivery rate 
of $1 \mu \mathrm{g} / \mathrm{kg} / \mathrm{min}$ over the course of 4 weeks as previously described (Cao et al., 2007). An AAA is defined as a 1.5-fold enlargement of the aortic diameter. Abdominal aortic enlargements, as well as aneurysm complications including aortic dissection and rupture were observed during the study. The Animal Use Committee at Queen's University approved all animal protocols.

\section{ULTRASOUND IMAGING ACOUISITION}

Ultrasound imaging was performed with mice placed supine on a heated table under isoflurane anesthesia and depilated with hair removal cream. A Vevo 770 high-resolution ultrasound imaging system (VisualSonics, Toronto, Canada) with $40 \mathrm{MHz}$ frequency real-time microvisualization scan-head (RMV 704) and $10 \times 10 \mathrm{~mm}$ field of view was used first in B-mode to obtain a 2D-transverse image to localize the suprarenal abdominal aorta on day 7 post-Ang II infusion. A more detailed examination of complex fluid-dynamical changes during AAA formation was obtained longitudinally by the ECG-based kilohertz visualization (EKV) reconstruction technique that synthesizes B-mode images from a series of heart rhythm cycles and reconstructs one representative heart cycle that is spatially precise and synchronized to the animal's ECG (VisualSonics software). All 65 mice with Ang II infusion were imaged over the 4-week course of the study. Power Doppler 3D-acquisition, which identifies blood flow inside the aortic lumen, was conducted among a set of 11 Ang II-infused mice on day 14 and 28. The probe mounted to the 3D motor stage was placed transversely on the abdomen covered with ultrasound gel to travel $10 \mathrm{~mm}$ along a suprarenal segment starting slightly above the right renal artery to obtain a series of 2D "slides", which were then assembled into a $3 \mathrm{D}$ data set for volume measurements. The ultrasound operator used manual assessment in delineation of the vessel and remodeled wall.

\section{AAA ASSESSMENT}

After mice were euthanized by $\mathrm{CO}_{2}$ asphyxiation at the day 28 endpoint, the aortic tree from the iliac bifurcation to aortic root was carefully dissected from surrounding tissue, and the abdominal aorta with or without aneurysm formation was measured and scored according to Daugherty's classification (Daugherty et al., 2001 ), then collected and embedded in OCT and stored at $-80^{\circ} \mathrm{C}$ until sections were cut for further histological and immunohistochemical analysis.

\section{HISTOLOGICAL AND IMMUNOFLUORESCENCE STAINING}

AAA sections were stained with Movat's pentachrome for morphology analysis. Inflammatory macrophages were labeled by immunofluorescence methods as described previously (Cao et al., 2007). Briefly, AAA sections were fixed with acetone for $5 \mathrm{~min}$, washed and blocked with 3\% normal goat serum for $30 \mathrm{~min}$, then incubated with primary antibodies against macrophage CD68 (1:100; Serotec, Oxford, UK) for $2 \mathrm{~h}$. After washing with PBS, specimens were incubated with fluorescently labeled secondary antibody Texas Red-conjugated goat serum (1:100; Jackson ImmunoResearch, West Grove, PA, USA) for $1 \mathrm{~h}$. Cover slips were mounted with VECTASHIELD plus DAPI (Vector, Burlingame, CA, USA). Visualization was performed with a fluorescent microscope (Leica, DM IRB, Richmond Hill, ON, USA). Quantification of $\mathrm{CD}^{+} 8^{+}$macrophages (relative to DAPI-stained cells) was conducted by Image-Pro Plus software (Media Cybernetics, Silver Spring, MD, USA).

\section{STATISTICAL ANALYSIS}

Data are expressed as mean \pm S.E.M. Differences between two groups were analyzed by Student's $t$-test. Correlation is tested by linear regression and Pearson coefficient analyses. Values of $P<0.05$ were considered significant.

\section{RESULTS \\ ANG II-INDUCED AORTIC RUPTURE OCCURS NOT ONLY IN THE ABDOMINAL AORTA BUT ALSO IN THE THORACIC REGION (AORTIC ARCH)}

In this study, $65 \mathrm{apoE}^{-/-}$male mice were implanted with Ang II pumps at a delivery rate of $1 \mu \mathrm{g} / \mathrm{kg} / \mathrm{min}$ for 4 weeks. Twenty-four mice (37\%) did not develop a detectable AAA according to standard criteria (i.e., 1.5-fold increase in suprarenal diameter), despite full release of Ang II from the pumps, while 41 mice (63\%) developed aortic aneurysms, with 25 surviving to the study endpoint (Table 1). Sixteen of the 41 mice died from aortic rupture (Table 2). By ultrasound scanning, we were able to detect 18 of the $25(72 \%)$ AAAs. However, seven small AAAs (28\%) went undetected because of their size ( $\approx 1.2 \mathrm{~mm}$; at the lower limit of being classified as an AAA), location (thoracic; not scanned) and orientation of dilatation. AAA severity, summarized according to Daugherty's classification (Daugherty et al., 2001) from 0 (no aneurysm) to IV (multiple aneurysms containing thrombus) plus the most severe situation - death from aortic rupture is shown in Table 2.

To examine aortic rupture incidence, we monitored each mouse intensively 7 days/week requesting animal care facility staff workers to report animal death immediately. Rapid detection of the first event enabled a thorough autopsy. No blood was found in the abdominal cavity ruling out an AAA rupture. However, careful dissection of the whole aortic tree revealed a blood clot in the chest cavity with an aortic arch rupture (Figure 1A). Later, we also found cases of abdominal aortic rupture (Figure 1B) and determined that most of the aortic ruptures occurred in the first week of Ang II infusion (Table 3). Surprisingly, ruptures at the arch were nearly as prevalent as in the suprarenal abdominal segment. All seven aortic ruptures at the arch region (100\%) were

Table 1 | Summary of Ang II-induced aneurysms in apoE-1- mice.

\begin{tabular}{lllll}
\hline $\begin{array}{l}\text { No of } \\
\text { mice } \\
\text { infused }\end{array}$ & $\begin{array}{l}\text { No of mice } \\
\text { with } \\
\text { aneurysms } \\
\text { (incidence) }\end{array}$ & $\begin{array}{l}\text { No of mice } \\
\text { with AAA to } \\
\text { endpoint (\%) }\end{array}$ & $\begin{array}{l}\text { No of AAA } \\
\text { detected by } \\
\text { echo (\%) }\end{array}$ & $\begin{array}{l}\text { No of AAA } \\
\text { not detected } \\
\text { by echo(\%) }\end{array}$ \\
\hline 65 & 41 & 25 & 18 & 7 \\
& $(63 \%)$ & $(61 \%)$ & $(72 \%)$ & $(28 \%)$
\end{tabular}

Table 2 | Classification of Ang II-induced aortic aneurysms.

\begin{tabular}{lllllll}
\hline Severity scale & $\mathbf{0}$ & I & II & III & IV & Rupture \\
\hline No of mice & 24 & 10 & 2 & 12 & 1 & 16
\end{tabular}




\section{Aortic arch rupture Abdominal aortic rupture}

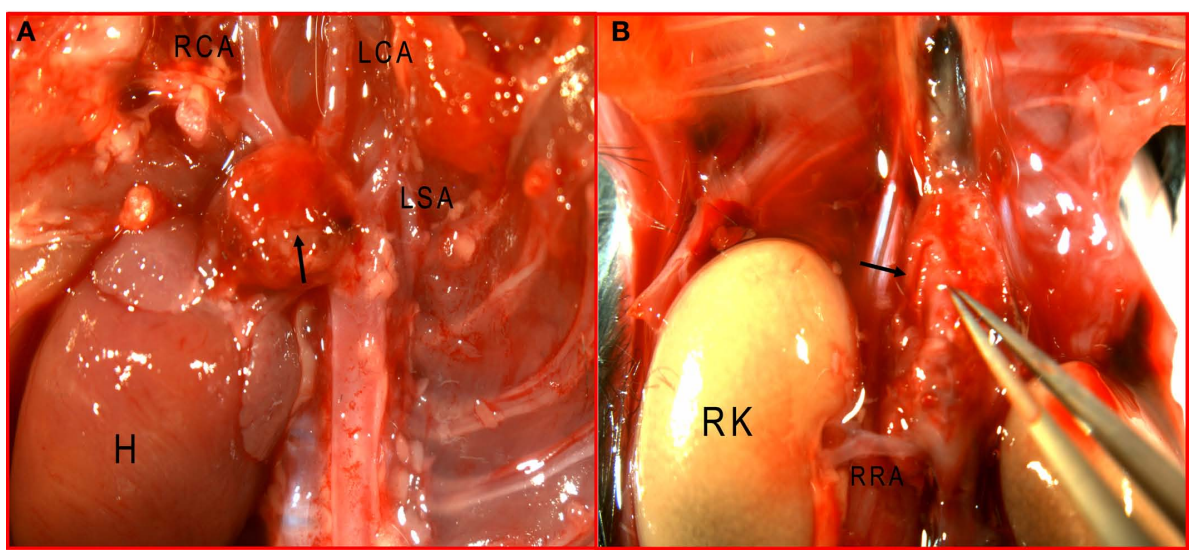

FIGURE 1 |Ang II induces aortic rupture not only in the abdominal aorta but also in the aortic arch. (A) Aortic arch, with major branches, was dissected to ascertain the exact rupture site; $(\mathbf{B})$ abdominal aorta was dissected from surrounding tissue to determine the suprarenal rupture site. Arrows indicate rupture sites. $\mathrm{H}$, heart; $\mathrm{RCA}$, right carotid artery; LCA, left common carotid artery; LSA, left subclavian artery; RK, right kidney, RRA, right renal artery.
Table 3 | Timing and site-specificity of Ang II-induced aortic rupture.

\begin{tabular}{lllllll}
\hline Time point/ & Week & Week & Week & Week & Abdominal & $\begin{array}{l}\text { Aortic } \\
\text { arch }\end{array}$ \\
\hline Nocation & $\mathbf{1}$ & $\mathbf{2}$ & $\mathbf{3}$ & $\mathbf{4}$ & & 7
\end{tabular}

fatal, while nine of the $34(26 \%)$ aneurysms in the abdominal aorta resulted in death with most showing signs of aortic dissection with subsequent remodeling by histological analysis (data not shown).

\section{AORTIC DISSECTION PRECEDES ALTERED FLOW PATTERNS WITHIN ANG II-INDUCED ANEURYSMAL AORTAS}

Using the Vevo 770 high-resolution ultrasound imaging system, we were able to detect AAAs in vivo and follow the mice longitudinally prior to sacrifice at day 28 . The transverse B-mode image applied to animals 7 days post-Ang II pump implantation, showed enlargement of the aorta (Figure 2A) more than $50 \%$ of the original diameter, while Power Doppler Mode imaging revealed blood flow within the aortic lumen (orangered; Figure 2B).

To elucidate flow pattern changes during aneurysm formation, abdominal aortas were visualized by B-mode longitudinally (Figure 3) and were recorded in the EKV-mode, which reconstructs B-mode images from a series of heart rhythm cycles into one representative heart cycle that is spatially precise and synchronized to the animal's ECG (Video S1 in Supplementary material). In the representative image, an aortic wall dissection is evident (white arrow, Figure 3). Circular flow patterns were obvious with a vortex pattern that sheds downstream (red lines, Figure 3; see Video S1 in Supplementary material). The vortex shedding has also been observed in numerical models of the fluid flow in murine AAA (Ford et al., 2010), where a vortex formed downstream of the aortic dissection "flap" during systolic acceleration is then advected into the aneurysm. A large recirculation zone that persists during the deceleration and diastolic tail of the cycle may be connected to the formation of a thrombus.

\section{ASSESSMENT OF ANEURYSM DIAMETER AND VOLUME BY ULTRASOUND IMAGING AND CORRELATION WITH HISTOLOGICAL PARAMETERS}

Measurements of aortic diameter were conducted via non-invasive ultrasound (Figures 4A,D), at endpoint dissection (Figures $4 \mathrm{~B}, \mathrm{E}$ ) and by histological analysis (Figures $4 \mathrm{C}, \mathrm{F}$ ). The results from the three different measurements correlate very well revealing a suprarenal diameter of $\approx 1 \mathrm{~mm}$ in Ang II-infused mice without aneurysm formation (Figure 4 , upper panels) and $\approx 2.8 \mathrm{~mm}$ with aortic aneurysm (Figure 4, lower panels). Correlation of aortic diameter measured by ultrasound and postmortem morphology was significant $\left(r^{2}=0.86, P<0.0001\right)$. A thrombus observed at the gross morphological level was further characterized by histological Movat's pentachrome staining to show remodeling events within the aortic wall where collagen is stained in yellow, ground substance and mucin in blue, fibrinoid and fibrin in intense red, and elastic fibers in black.

Previously AAA complexity was determined by Daugherty's classification (Daugherty et al., 2001), by measurement of the aortic diameter (Cao et al., 2007; Gitlin et al., 2007) or by AAA weight assessment (King et al., 2006; Gitlin et al., 2007; Wang et al., 2008). To better reveal changes during AAA formation, we conducted Power Doppler 3D-acquisition on day 14 and 28 on 11 mice postAng II infusion (five without AAA and six with AAA) to obtain $3 \mathrm{D}$ geometry parameters, which were further reconstructed into volume measurements. We found, as expected, AAA volumes were significantly greater than measurements obtained from the same suprarenal aortic segment without AAA formation (Figures 5A,B) and correlate with AAA diameter $\left(r^{2}=0.81, P<0.001\right.$, not shown) and strongly correlates with AAA severity (Figure 5C). Therefore, AAA volume could be used as a new parameter to indicate AAA 


\section{Transverse B Mode}

Power Doppler Mode

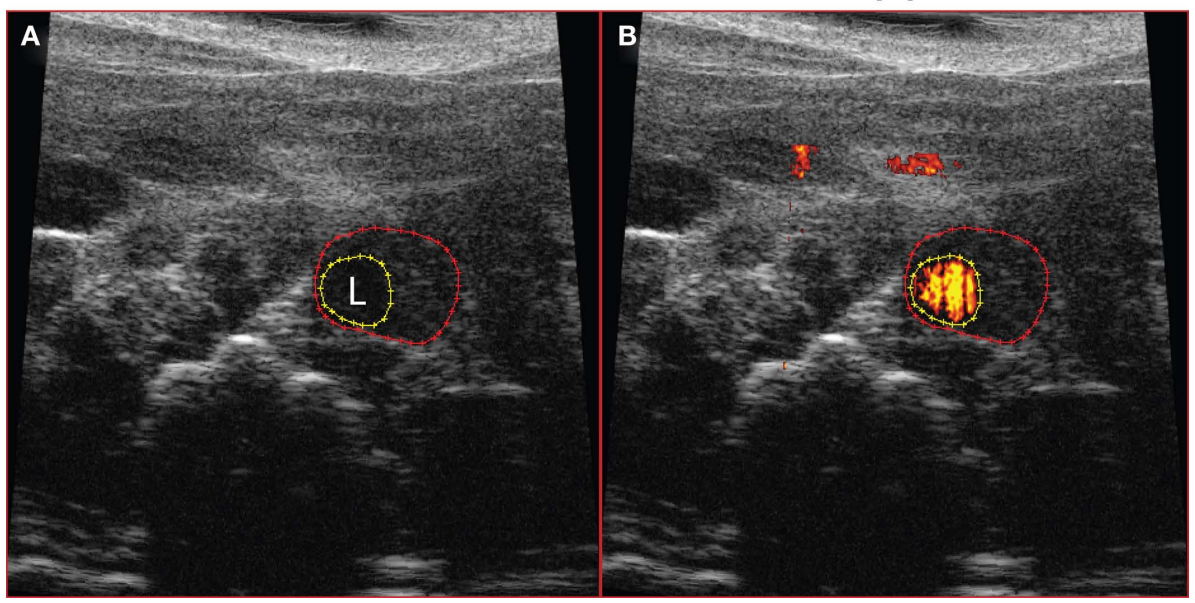

FIGURE 2 |Abdominal aortic dilatation was detected by ultrasound imaging 7 days post-Ang II infusion. (A) Transverse B-mode shows enlargement and remodeling within the aortic wall; (B) Power Doppler Mode demonstrating blood flow in the aortic lumen. Dotted red line outlines external aortic boundary, while the yellow dotted line outlines the lumen $(\mathrm{L})$.

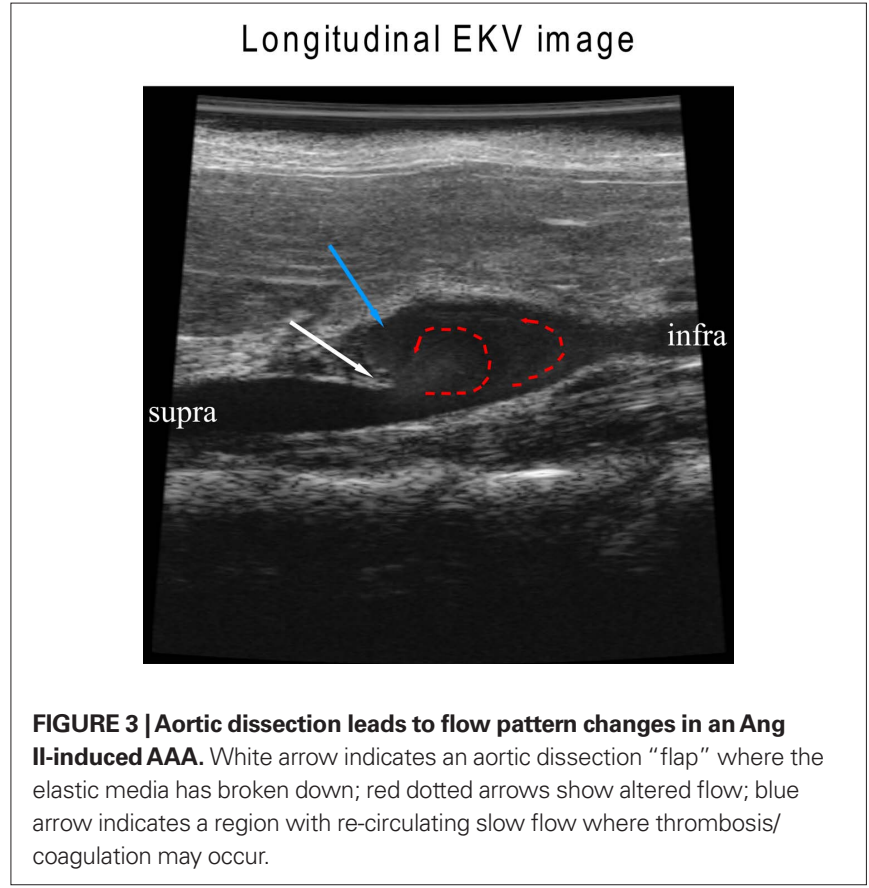

severity. Moreover, 3D rendering enables visualization from different angles and planes for more detailed analyses (Video S2 in Supplementary material).

\section{MACROPHAGES ARE MAJOR INFLAMMATORY CELLS IN ANEURYSMAL TISSUE}

To evaluate inflammatory cell infiltration within aneurysmal tissue, aortic specimens were sectioned and inflammatory components were labeled with immunofluorescent antibodies. We found few $\mathrm{T}$ cells and mast cells (data not shown) but large numbers of CD68 ${ }^{+}$ macrophages in the adventitia (Figures 6A,B), especially at the site of elastic media break down (Figures 6C,D), indicating an important role of macrophages in aortic aneurysm development.

\section{DISCUSSION}

The Ang II-induced mouse model of AAA is characterized by macrophage infiltration, elastic media degeneration, aortic dissection, thrombus formation, aneurysmal wall remodeling, and aortic rupture (Daugherty et al., 2000; Saraff et al., 2003; Cao et al., 2007). Here, we have carried out a detailed time-frame and site-specific analysis of aortic rupture combined with ultrasound imaging of aneurysms.

In humans, about $80 \%$ of aortic aneurysms occur in the abdominal region (Baxter et al., 2008). The well-established murine AAA model used here by us and many other investigators shares several common features with human AAAs as mentioned above, as well as male gender preponderance, hypertension, and hyperlipidemia (apoE $\mathrm{E}^{-/-}$mice). There are, however, certain differences. For instance, in mice these aneurysms occur most frequently in the suprarenal aorta as opposed to the infrarenal location in humans. Moreover, aortic dissection and rupture are early events in the mouse model in the absence of atherosclerosis, in contrast to the complications of dissection and rupture that tend to occur in late-stage, large aneurysms with manifest atherosclerosis in humans.

Aortic rupture is a fatal complication of both human and mouse AAAs. Death-associated aortic rupture was only reported at $\approx 10 \%$ when this Ang II-induced AAA model was first established (Saraff et al., 2003). Recently, high rupture mortality was reported in apoE ${ }^{-/}$ mice (47\%) (Chamberlain et al., 2010) and in IFN- $\gamma /$ apoE double knockout mice (50\%) (King et al., 2008). In our study we observed $25 \%$ (16 of 65) aortic rupture deaths in apoE $\mathrm{E}^{-/-}$mice and found that most ruptures happened in the first week of Ang II infusion (12 of 16; Table 3). Interestingly, we found that rupture was almost as common in the aortic arch as it was in the suprarenal abdominal 


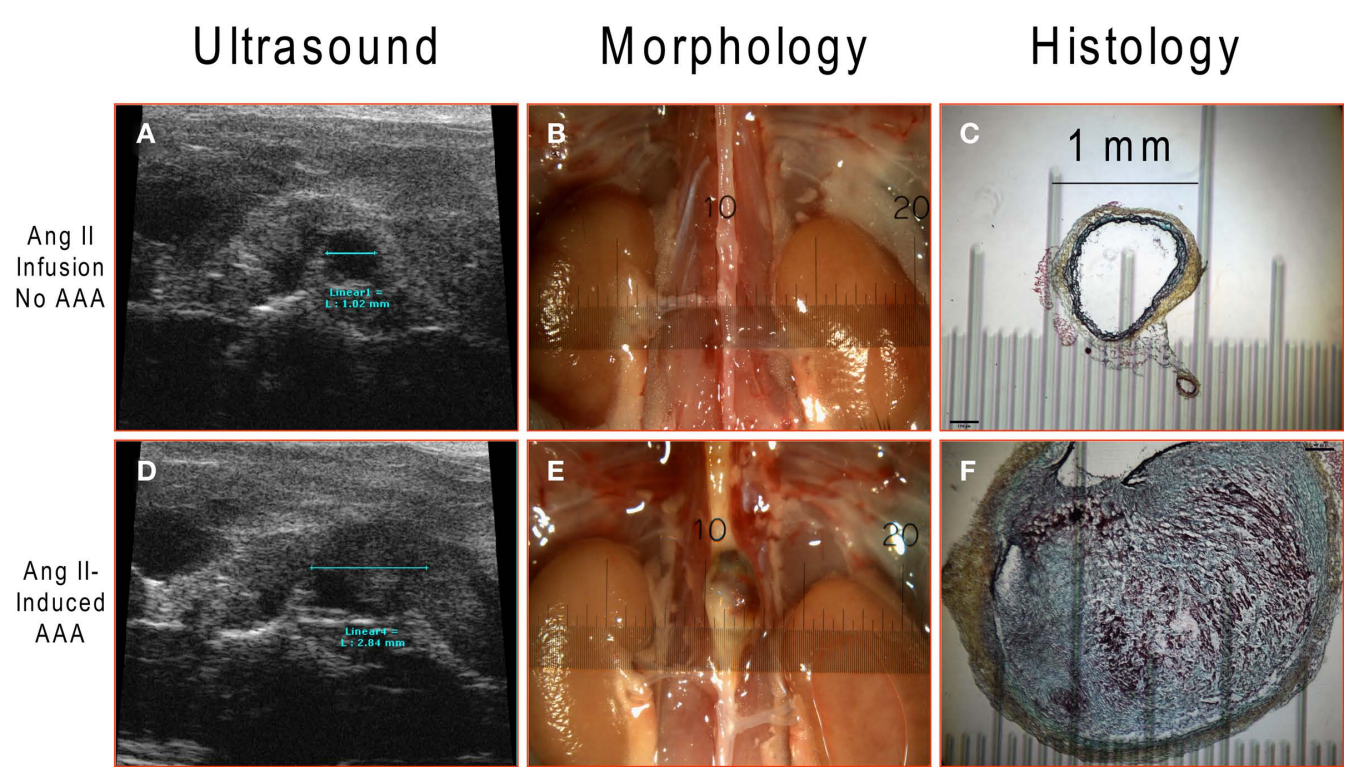

FIGURE 4 | Ang Il-induced AAA detected by ultrasound imaging and further verified by dissection and histology. A representative abdominal aorta without AAA formation (upper panels; $n=24$ ) and with AAA (lower panels; $n=25)$ after 4-week Ang II infusion: $(\mathbf{A}, \mathbf{D})$ Ultrasound images; (B,E) gross morphological view; (C,F) Movat's pentachrome histological staining.

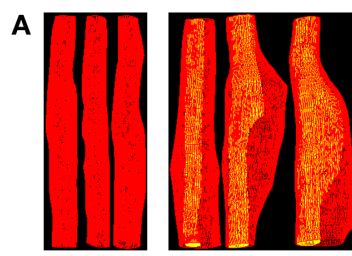

Ang II no AAA Ang II AAA

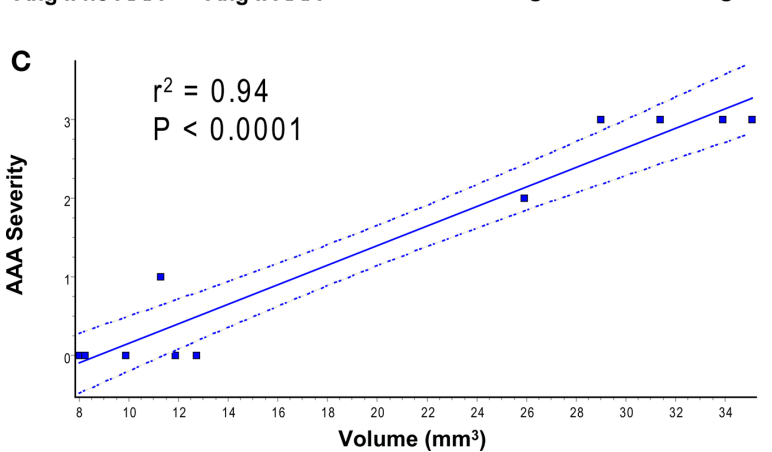

FIGURE 5 |Ang II-induced AAA volume measurements correlate with AAA severity. (A) Reconstructed volumes of abdominal aorta without (left panel of three representative aortas) and with AAA formation (right panel of three representative aortas). (B) Quantification of volume in a landmarked suprarenal segment. ${ }^{*} P<0.01$ between Ang II infusion without AAA formation ( $n=5$ ) and with Ang II-induced AAA formation $(n=6)$. (C) Linear regression analysis shows strong correlation between aortic 3D volume and Daugherty's AAA severity classification $(P<0.0001)$. The dotted lines represent $95 \%$ confidence intervals.

site, where aneurysms usually develop in this model (Figure 1). On one occasion, rupture occurred during the ultrasound scanning procedure indicating that pressure from the scan-head probe on the body surface could be sufficient to trigger AAA rupture.
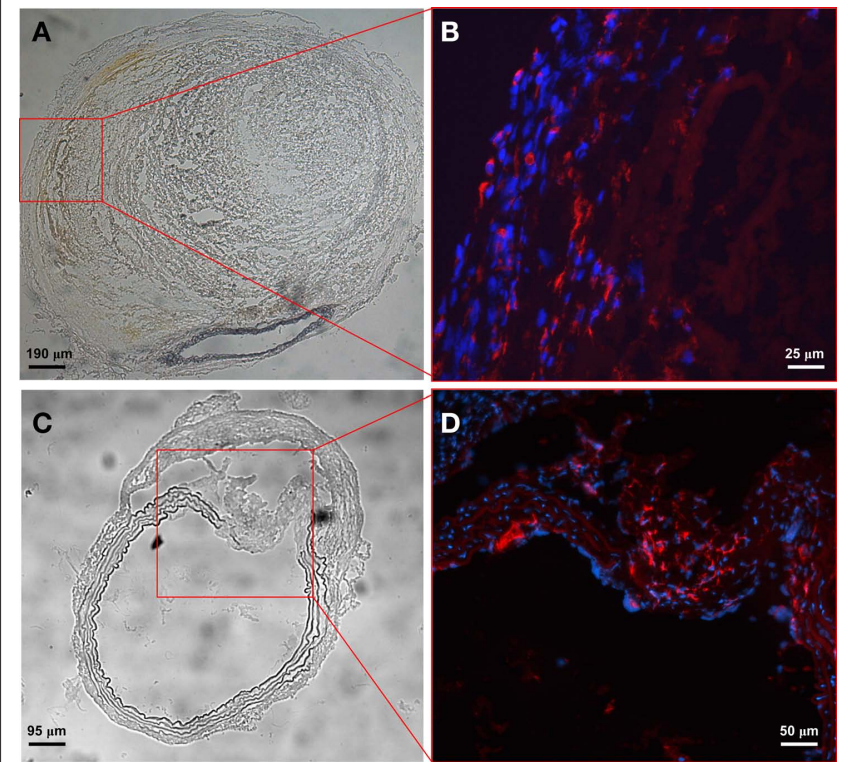

FIGURE 6 | Macrophages are major inflammatory cells in aneurysmal tissue. Phase contrast images of a severely remodeled AAA (A) and one with extensive elastic layer damage (C). Enhanced images from the boxed area show predominant $\mathrm{CD}_{6} 8^{+}$macrophages in the adventitia (B) and macrophage infiltration at the site of elastic media break down (D). The percentage of $\mathrm{CD}^{+} 8^{+}$macrophages (relative to DAPI-stained cells) is $42 \%$ in the boxed area $(\mathbf{C}, \mathbf{D})$ where the elastic media shows evidence of breakdown and only $3 \%$ in the remaining area.

Previously, flow patterns and fluid dynamics modeling have been reported in human AAAs (Castro et al., 2006; Baek et al., 2010; Les et al., 2010), with very little insight into patterns in mice. In the present study, we were able to monitor the 
progression of AAA in this mouse model using non-invasive, high-resolution ultrasound specially designed for small animals. This imaging system not only allowed us to assess aortic diameter (Figure 4), which significantly correlated with postmortem morphology aortic measurement $\left(r^{2}=0.86, P<0.0001\right)$ and $3 \mathrm{D}$ geometry (Figure 5), which Goldberg et al. (2007) reported to have strong correlation with histology volumes reconstructed by aligning each $2 \mathrm{D}$ digitized section. The imaging also allowed us to dynamically monitor flow patterns during AAA formation at three time-points. One limitation to this methodology is that more frequent, repeated exams necessitates multiple periods of deep isoflurane anesthesia and depilation for scan-head contact, which can lead to altered mouse physiology and skin lesions. So, we have opted for analysis at weekly intervals. In a previous report, aortic expansion induced by Ang II infusion was detected by ultrasound (Barisione et al., 2006), but in our present study details of complex fluid flow patterns were observed and were already evident at day 7 post-Ang II infusion with unsteady flow, shed vortices that impinge upon the distal wall, and re-circulating flow (Figure 3). Using a computational fluid dynamics model, Baek et al. (2009) found the impingement region coincides with the location of rupture. Thus, if the repair process, which includes thrombus formation/coagulation/fibrosis in a dynamic remodeling fashion cannot override the degenerative process after aortic dissection at the intimal layer, then adventitial weakening will inevitably lead to aortic rupture with subsequent hemorrhage and death.

The role of fluid mechanics in the formation of the AAA remains an open question. Although the aneurysms studied here develop from Ang II infusion via an initial dissection in the intimal wall, once formed the local fluid mechanics can play a critical role in the growth and subsequent rupture of the AAA. In our studies, there is an area in which the local fluid mechanics are favorable to the formation of thrombus (slow recirculation zone above the distal entrance to the aneurysm at the dissection) and hence growth of the vessel wall. The impingement of the shed vortex on the distal end of the aneurysm may also give rise to an

\section{REFERENCES}

Baek, H., Jayaraman, M. V., and Karniadakis, G. E. (2009). Wall shear stress and pressure distribution on aneurysms and infundibulae in the posterior communicating artery bifurcation. Ann. Biomed. Eng. 37, 2469-2487.

Baek, H., Jayaraman, M. V., Richardson, P. D., and Karniadakis, G. E. (2010). Flow instability and wall shear stress variation in intracranial aneurysms. $J$. R. Soc. Interface 7, 967-988.

Barisione, C., Charnigo, R., Howatt, D. A., Moorleghen, J. J., Rateri, D. L., and Daugherty,A. (2006). Rapid dilation of the abdominal aorta during infusion of angiotensin II detected by noninvasive high-frequency ultrasonography. J. Vasc. Surg. 44, 372-376.

Baxter, B. T., Terrin, M. C., and Dalman, R. L. (2008). Medical management of area susceptible to expansion or even rupture. In future studies we aim to explore the role of the fluid mechanics on the rupture risk of mouse AAA.

Inflammation is commonly recognized to be the key to aneurysmal diseases (Wassef et al., 2007; Norman and Powell, 2010). In our study, we found prominent inflammatory macrophage infiltration at the site of elastic media break down (Figure 6). Tieu et al. (2009) found that an IL-6/MCP-1 amplification loop accelerates macrophage-mediated vascular inflammation leading to aortic dissection in mice, while others (King et al., 2008; Wang et al., 2010) found TGF- $\beta$ and interferon- $\gamma$ to protect against inflammatory aortic aneurysm progression. Therefore, pharmacological intervention to block inflammatory pathways may prevent the progression of AAAs. Currently, there are no extant therapeutics for blocking AAA progression although agents such as statins, doxycycline, matrix metalloproteinase inhibitors, cyclooxygenase (COX)-2 inhibitors, and angiotensin receptor antagonists have all been shown to modulate the progression of aortic aneurysms in murine models (Wassef et al., 2007). The selective COX-2 inhibitor celecoxib has shown efficacy to decrease Ang II-induced mouse AAAs (King et al., 2006), but this class of drugs enhances cardiovascular risk in humans. To avoid such hazard, we are currently investigating downstream prostanoid receptor antagonists to target attenuation of AAA progression.

\section{ACKNOWLEDGMENTS}

This work was supported by CIHR grant MOP-93689, Heart and Stroke Foundation of Ontario Career Investigator Award (CI5960), the Canada Research Chairs program, Canadian Foundation for Innovation and Ministry of Research and Innovation Ontario. The authors would like to thank Jeffrey Mewburn and Jalna Meens for helpful assistance with the Visualsonics Vevo770 ultrasound imaging.

\section{SUPPLEMENTARY MATERIAL}

The Supplementary Material for this article can be found online at http://www.frontiersin.org/Pharmacology/Pharmacotherapy of Inflammation/paper/10.3389/fphar.2010.00009/
(2010). Perforin-independent extracellular granzyme B activity contributes to abdominal aortic aneurysm. Am. J. Pathol. 176, 1038-1049.

Daugherty, A., and Cassis, L. A. (2004). Mouse models of abdominal aortic aneurysms. Arterioscler. Thromb. Vasc. Biol. 24, 429-434.

Daugherty,A.,Manning,M.W., and Cassis, L. A. (2000). Angiotensin II promotes atherosclerotic lesions and aneurysms in apolipoprotein e-deficient mice. $J$. Clin. Invest. 105, 1605-1612.

Daugherty, A., Manning, M. W., and Cassis, L. A. (2001). Antagonism of AT2 receptors augments angiotensin II-induced abdominal aortic aneurysms and atherosclerosis. $\mathrm{Br}$. J. Pharmacol. 134, 865-870.

Daugherty, A., Rateri, D. L., and Cassis, L. A. (2006). Role of the reninangiotensin system in the development of abdominal aortic aneurysms in animals and humans. Ann. N.Y. Acad. Sci. 1085, 82-91.

Ford, M. D., Piomelli, U., Cao, R. Y., Funk, C. D., and Pollard, A. (2010). "Numerical simulations of the intra-aneurysmal vortex shedding in induced mouse abdominal aortic aneurysms," in Proc. ASME 2010 3rd Joint US-European Fluids Eng. Summer Meeting and 8th International Conf. on Nanochannels, Microchannels, and Minichannels, FEDSM-ICNMM201030546.

Gitlin, J. M., Trivedi, D. B., Langenbach, R., and Loftin, C. D. (2007). Genetic deficiency of cyclooxynenase- 2 attenuates abdominal aortic aneurysm formation in mice. Cardiovasc. Res. 73, 227-236.

Goldberg, A., Pakkiri, P., Dai, E., Lucas, A., and Fenster, A. (2007). Measurements 
of aneurysm morphology determined by $3-\mathrm{D}$ micro-ultrasound imaging as potential quantitative biomarkers in a mouse aneurysm model. Ultrasound Med. Biol. 33, 1552-1560.

Golledge, J., Muller, J., Daugherty, A., and Norman, P. (2006). Abdominal aortic aneurysm: pathogenesis and implications for management. Arterioscler. Thromb. Vasc. Biol. 26, 2605-2613.

King, V.L., Lin, A. Y., Kristo, F., Anderson, T. J.T., Ahluwalia, N., Hardy, G. J., Owens, A.P.III., Howatt, D. A., Shen, D., Tager, A.M., Luster, A. D., Daugherty, A., and Gerszten, R. E. (2008). Interferon- $\gamma$ and the interferon-inducible chemokine CXCL10 protect against aneurysm formation and rupture. Circulation 119, 426-435.

King, V.L., Trivedi, D. B., Gitlin, J. M., and Loftin, C. D. (2006). Selective cyclooxygenase-2 inhibition with celecoxib decreases angiotensin II-induced abdominal aortic aneurysm formation in mice. Arterioscler. Thromb. Vasc. Biol. 26, 1137-1143.

Les, A. S., Shadden, S. C., Figueroa, C. A., Park, J. M., Tedesco, M. M., Herfkens, R. J., Dalman, R. L., and Taylor, C. A. (2010). Quantification of hemodynamics in abdominal aortic aneurysms during rest and exercise using magnetic resonance imaging and computational fluid dynamics. Ann. Biomed. Eng. 38, 1288-1313.
Lloyd-Jones, D., Adams, R. J., Brown, T. M., Carnethon, M., Dai, S., De Simone, G., Ferguson, T. B., Ford, E., Furie, K., Gillespie, C., Go, A., Greenlund, K., Haase, N., Hailpern, S., Ho, P. M., Howard, V., Kissela, B., Kittner, S., Lackland, D., Lisabeth, L., Marelli,A., McDermott, M. M., Meigs, J., Mozaffarian, D., Mussolino, M., Nichol, G., Roger, V.L., Rosamond, W., Sacco, R., Sorlie, P., Stafford, R., Thom, T., Wasserthiel-Smoller, S., Wong, N. D., and Wylie-Rosett, J. (2010). Heart disease and stroke statistics - 2010 update: a report from the American Heat Association. Circulation 121, e46-e215.

McCormick, M. L., Gavrila, D., and Weintraub, N. L. (2007). Role of oxidative stress in the pathogenesis of abdominal aortic aneurysms. Arterioscler. Thromb. Vasc. Biol. 27, 461-469.

Norman, P. E., and Powell, J. T. (2010). Site specificity of aneurysmal disease. Circulation 121, 560-568.

Saraff, K., Babamusta, F., Cassis, L. A., and Daugherty, A. (2003). Aortic dissection precedes formation of aneurysms and atherosclerosis in angiotensin II-infused apolipoprotein E-deficient mice. Arterioscler. Thromb. Vasc. Biol. 23, 1621-1626.

Thompson, R. W. (2002). Reflections on the pathogenesis of abdominal aortic aneurysms. Cardiovasc. Surg. 10, 389-394.

Tieu, B. C., Lee, C., Sun, H., LeJeune, W., Recinos, A., 3rd., Ju, X., Spratt, H., Guo, D., Milewicz, D., Tilton, R. G., and Brasier, A. R. (2009). An adventitial IL-6/MCP1 amplification loop accelerates macrophages-mediated vascular inflammation leading to aortic dissection in mice. J. Clin. Invest. 119, 3637-3651.

Upchurch, G. R. Jr., and Schaub, T. A. (2006). Abdominal aortic aneurysm. Am. Fam. Physician 73, 1198-1204.

van Vlijmen-van Keulen, C. J., Pals, G. and Rauwerda, J. A. (2002). Familial abdominal aortic aneurysm. Eur. J. Vasc. Endovasc. Surg. 24, 105-116.

Wang, M., Lee, E., Song, W., Ricciotti, E., Rader, D. J., Lawson, J.A., Pure, E., and FitzGerald, G. A. (2008). Microsomal prostaglandin E synthase-1 deletion suppresses oxidative stress and angiotensin II-induced abdominal aortic aneurysm formation. Circulation 117 1302-1309.

Wang, Y., Ait-Oufella, H., Herbin, O., Bonnin, P., Ramkhelawon, B., Taleb, S. Huang, J., Offenstadt, G., Combadiere, C., Renia, L., Johnson, J. L., Tharaux, P., Tedgui,A., and Mallat,Z. (2010). TGF- $\beta$ activity protects against inflammatory aortic aneurysm progression and complications in angiotensin II-infused mice. J. Clin. Invest. 120, 422-432.
Wassef, M., Upchurch, G. R., Jr. Kuivaniemi, H., Thompson, R.W., and Tilson, M. D. III. (2007). Challenges and opportunities in abdominal aortic aneurysm research. J. Vasc. Surg. 45, 192-198.

Conflict of Interest Statement: The authors declare that the research was conducted in the absence of any commercial or financial relationships that could be construed as a potential conflict of interest.

Received: 10 May 2010; paper pending published: 26 May 2010; accepted: 22 June 2010; published online: 14 July 2010.

Citation: Cao RY, St. Amand T, Ford MD, Piomelli $U$ and Funk CD (2010) The murine angiotensin II-induced abdominal aortic aneurysm model: rupture risk and inflammatory progression patterns. Front. Pharmacol. 1:9. doi: 10.3389/ fphar.2010.00009

This article was submitted to Frontiers in Inflammation Pharmacology, a specialty of Frontiers in Pharmacology.

Copyright (C) 2010 Cao, St. Amand, Ford, Piomelli and Funk. This is an open-access article subject to an exclusive license agreement between the authors and the Frontiers Research Foundation, which permits unrestricted use, distribution, and reproduction in any medium, provided the original authors and source are credited. 